\title{
ANÁlisis DE CICLO DE VIDA PARA UNA BIOREFINERÍA DERIVADA DE RESIDUOS AGRÍCOLAS DE PALMA ACEITERA (ELAEIS GUINEENSIS)
}

\author{
LIFE CYCLE ANALYSIS FOR A BIOREFINERY FROM \\ OIL PALM FRONDS (Elaeis guineensis)
}

\section{${ }^{1}$ Jesús A. Torres Ortega, ${ }^{2}$ Oscar F. Contento Rubio, ${ }^{3}$ Israel Herrera Orozco}

${ }^{1,2}$ Universidad de La Salle, Programa de Ingeniería Ambiental y Sanitaria. Bogotá, Colombia ${ }^{3}$ Centro de Investigaciones Energéticas, Medio Ambientales y Tecnológicas - Ciemat, Departamento de Energía. Madrid, España.

\footnotetext{
1jatorres@unisalle.edu.co, orcid.org/0000-0002-4476-0816

20fcontento@unisalle.edu.co, orcid.org/0000-0002-2958-9491

${ }^{3}$ israel.herreradciemat.es, orcid.org/0000-0003-1864152X
}

\begin{abstract}
RESUMEN
La palma aceitera colombiana es un producto agrícola no alimenticio es importante para la economía del pais debido a su mercadeo en el extranjero. Lo cual se refleja en que el volumen de producción colocó a Colombia como el sexto exportador de aceite de palma en el mundo. Las plantas extractoras producen casi 1.6 toneladas de biomasa húmeda concentrada por tonelada de producto primario comercializado (aceite y almendra), adicionalmente de 0.7 metros cúbicos de efluente líquido por tonelada de racimos de fruta fresca es obtenido. Este estudio nos permite caracterizar la cantidad de residuos utilizados en las extractoras de aceite de palma como un escenario de muestra representativa para identificar los posibles impactos potenciales para las opciones de su diversificación industrial. Actualmente, la idea de una economía circular tiene un papel protagónico en las agendas comerciales mundiales al punto de desvincular el crecimiento económico de las limitaciones de recursos, por cuanto busca maximizar el valor en cada punto de la vida de un producto. Esta revisión bibliográfica tiene como propósito referenciar un desarrollo metodológico de la sostenibilidad del ciclo de vida para respaldar la valoración de estrategias de economía circular en un escenario 'cradle to cradle' de la cuna a la cuna para un caso de estudio como primera entrega de la visualización del inventario para los residuos agricolas de un cultivo de palma aceitera. Para la investigación se propone usar el software SimaPro que ayuda en la selección de los criterios para considerar en el proceso de extracción y posterior transformación y recirculación de los desechos agrícolas asi como de los biocombustibles generados mediante las bases de datos Ecoinvent, lo que permitirá establecer indicadores para una economía circular que sea sostenible mediante estrategias de circularidad, es decir, opciones que apuntan a mantener los residuos, componentes y materiales en su máxima utilidad en todo momento del Análisis del Ciclo de Vida (ACV) para permitir una gestión eficiente de recursos, nutrientes y agroquimicos, estos parámetros permiten identificar aquellos elementos puedan utilizar alternativas para reducir los requisitos
\end{abstract}


y posibilitar la sostenibilidad del inventario del cultivo de la palma aceitera. El objetivo de la investigación en curso es evaluar el ACV en un cultivo representativo de la palma aceitera colombiana, para dar cuenta de los suministros de la agricultura y el consumo en una muestra representativa del cultivo como auto generadora de combustibles. Asi mismo, se pretende aportar información en este ámbito para la futura toma de decisiones hacia la sostenibilidad de los recursos y la optimización de los procesos por parte de las empresas de cultivadores de palma como parte de su politica de responsabilidad ambiental.

Palabras clave: aceite, hidrólisis, impactos, refinería, desperdicios.

\section{Abstract}

The cultivation of Colombian palm is a non-food agricultural product important for the economy due to its market abroad. The volume of production placed Colombia as the sixth exporter of palm oil in the world. The extraction plants produce almost 1.6 tons of concentrated wet biomass per ton of marketed primary product (oil and almond), additionally 0.7 cubic meters of liquid effluent per ton of fresh fruit bunches is obtained. This study allows us to characterize the amount of waste used in the palm oil refinery as a representative sample scenario to identify potential impacts for industrial diversification options. Currently, the idea of a circulate economy plays a leading role in world trade agendas to the point of decoupling economic growth from resource constraints, it seeks to maximize value at every point in the life of a product. This review aims to present a methodological development and the applications of independent or integrated life cycle approaches: environmental footprint - life cycle sustainability assessment to support the valuation of circular economy strategies in a scenario 'cradle to cradle'. SimaPro ${ }^{\circ}$ Designer is software used for the research that help to select the criteria to be considered in the process of extraction and subsequent transformation and recirculation of the agricultural waste as well as the biofuels generated through the Ecoinvent databases, which will allow to establish indicators for a circular economy that is sustainable through circularity strategies, that is, options that aim to keep waste, components and materials at their maximum use at all times such as life cycle analysis (LCA) to allow effcient management of resources, nutrients and agrochemicals, knowing that these parameters allow us to identify those elements that influence its magnitude, so that they can use alternatives to reduce the requirements and make possible the sustainability of the oil palm crop inventory. The aim research to evaluate LCA of the Colombian palm oil crop representative for account for agricultural supplies and consumption as case sample of energy efficient by the company. Likewise, provide information in this field for future decision making towards the sustainability of resources and the optimization of processes by companies of palm growers as part of their environmental responsibility policy.

Keywords: hydrolysis, impact, refinery, oil, remaining.

\section{INTRODUCCIÓN}

La palma es un producto agrícola no alimenticio importante para la economía colombiana debido a su comercialización en el exterior. Tanto así que en el país hay 450.131 ha cultivadas con palma de aceite, los rendimientos de los cultivos comerciales de palmas $E$. guineensis adultas oscilan entre 21 y 30 toneladas de racimos de fruta fresca (RFF) por hectárea, mientras que el correspondiente a los cultivos $\mathrm{OxG}$ oscila entre 22 y $32 \mathrm{t} \mathrm{RFF/ha.} \mathrm{El} \mathrm{costo} \mathrm{promedio} \mathrm{por} \mathrm{tonelada} \mathrm{de} \mathrm{RFF}$ en 2014 era $\$ 247.181$ para cultivos de E. guineensis y de \$251.798 para cultivos OxG, el costo por tonelada de aceite de palma crudo (APC) fue \$1'365.607 para cultivos de E. guineensis y $\$ 1$ '417.923 para $\mathrm{OxG}$ (Mosquera et al., 2016). La huella hídrica generada por 
la producción de este sector asciende a unos 2000 metros cúbicos por tonelada de palma de fruta, creando un problema potencial de seguridad alimentaria y disponibilidad de recursos para las generaciones futuras (Arévalo, 2012; Asociación española para la calidad, 2014). A partir de esto, surge el concepto de Análisis de Ciclo de vida, agregando criterios para evaluar el sector agrícola. Esto ha provocado la necesidad de establecer indicadores que permitan una gestión eficiente de los recursos disponibles, que permita cuantificar las cantidades requeridas para producir un determinado producto. Sabiendo que estos parámetros permiten identificar aquellos elementos que influyen en su magnitud, para que puedan usar alternativas para reducir los requerimientos de insumos de un cultivo en particular. El objetivo es aportar información en este ámbito para la futura toma de decisiones hacia la sostenibilidad de las plantas extractoras y la optimización de procesos por parte de las empresas cultivadoras de palma como parte de su política de responsabilidad ambiental. La transformación de las plantas extractoras existentes a biorefinerías podría ayudar a mejorar la competitividad de la industria palmera (Arrieta et al., 2007; Shuit et al., 2009; Vijayendran, 2010).

Actualmente se obtiene biodiesel y otros productos oleoquímicos a partir de los aceites de palma y de palmiste (Torres, 2012), a la vez que se investiga sobre bioetanol, productos químicos y otros tipos de biocombustibles. También se puede producir químicos de alto valor y biogás a partir de los efluentes de las plantas de beneficio. La diversificación de la industria palmera debe conducir a la reingeniería de los desechos agroindustriales que en su gran mayoría es biomasa utilizando la infraestructura de la planta lo cual es una gran oportunidad que traerá beneficios económicos, ambientales y sociales que se deberán analizar a corto plazo (Martínez, 2017). Este trabajo busca revisar diferentes escenarios para la conversión de las plantas extractoras en biorefinerías. En este sentido desarrollar una metodología que permita comparar diversas alternativas de uso de los residuos en la extracción del aceite contribuirá al sector de la palma aceitera a identificarse e implementar los conceptos de sostenibilidad e interiorizar consideraciones ambientales en los procesos que implementen.
El grupo de investigación en Gestión del Riesgo y Cambio Climático (GrYCC) Centro de Investigaciones Energéticas, Medioambientales y Tecnológicas (Ciemat) ven la oportunidad de optimizar la funcionalidad de sus espacios, además de plantear una metodología ACV experimental a nivel de planta piloto, donde se desarrollen las etapas de fermentación y destilación, a partir de residuos lignocelulósicos agrícolas que hayan sido preparados vía hidrólisis (ácida, básica, enzimática o sub-crítica) para ser fermentados y destilados en la planta, con el objeto de obtener un bioalcohol (González \& Neira, 2017; Herrera, 2016). Se busca promover la investigación en el campo de la generación de biocombustibles como fuente de eficiencia energética alterna a nivel académico e industrial. Como resultados previos y que dan origen a la formulación de la propuesta de innovación, el equipo de investigación cuenta por parte del investigador Israel Herrera Orozco (Сіемат) con la siguiente producción: Mercado \& Herrera (2017); Muñoz \& Herrera (2017); Vides et al. (2017); Herrera et al. (2016a); Muñoz et al. (2016); Herrera et al. (2016b); Gamarra et al. (2016). Por otra parte Jesús Torres (Unisalle), ha sido coinvestigador de los siguientes proyectos de investigación ya culminados: Obtención de esteres surfactantes derivados del aceite de palma (Colciencias); Instalación, puesta en marcha y ajuste tecnológico de un sistema piloto de producción de alcohol carburante a pequeña escala a partir de la caña de azúcar en hoya del río Suárez (Corpoica); Estudio de factibilidad técnica y económica de la producción de biodiesel a pequeña escala a partir de aceite de palma (Elaeis Guineensis), Noli (Elaeis Oleifera), Noli x Palma (E. Oleifera x E. Güineensis) y de palmáceas nativas del pacifico colombiano (Corpoica); Nuevo método para la investigación de procesos cataliticos (Colciencias); Determinación de la huella hidrica para cultivos de la agroindustria nacional (Unisalle). Cabe esperar que trabajos similares puedan extrapolarse a otros gremios agroindustriales en un mediano plazo por cuanto ya se han realizado trabajos similares en otros sectores agrícolas como flores, hortalizas, vegetales y otros: Torres et al. (2009 a,b); Torres (2010); Torres \& Maldonado (2011); Torres (2012); Torres (2014); Torres et al. (2015); Torres et al. (2016); así como la generación de propuestas con alternativas sostenibles para la agroindustria: 
Barón (2015); Bello \& Castellanos (2016); Carrero \& Loza (2016); Castañeda y Ramírez (2016); Deaza \& Godoy (2016); Garzón \& Pacheco (2016); Mora \& Mendoza (2017); Nieto \& Piñeros (2016); Pinzón \& Palacios (2016).

La anterior producción permite establecer la evolución del concepto de sostenibilidad en términos de la distribución de los excedentes de la producción agrícola. Esta perspectiva conduce a la necesidad de identificar las relaciones de equidad y comercio justo, necesarios para garantizar el desarrollo de prácticas agrícolas sustentables. Entonces, sugerir aspectos relevantes para la formulación de tecnologías emergentes con enfoque diferencial, mediante la integración de actores novedosos como el uso de bioresiduos, el acceso a recursos y knowhow, conlleva a una estructuración de las cadenas de valor (Basiron y Darus, 1997; García et al., 2010).

\section{Procesamiento de los Racimos DE FRUTA FRESCA (RFF)}

La composición de un racimo de palma aceitera se presenta en la Fig. 1. Luego de la recolección y almacenamiento del fruto, el racimo pasa a un proceso de esterilización, luego se descarta el fruto de su raquis para conducirlo a los digestores. El objeto del digestor es acondicionar el fruto, haciendo que se aparte el mesocarpio de las nueces para facilitar la extracción del aceite en el prensado.
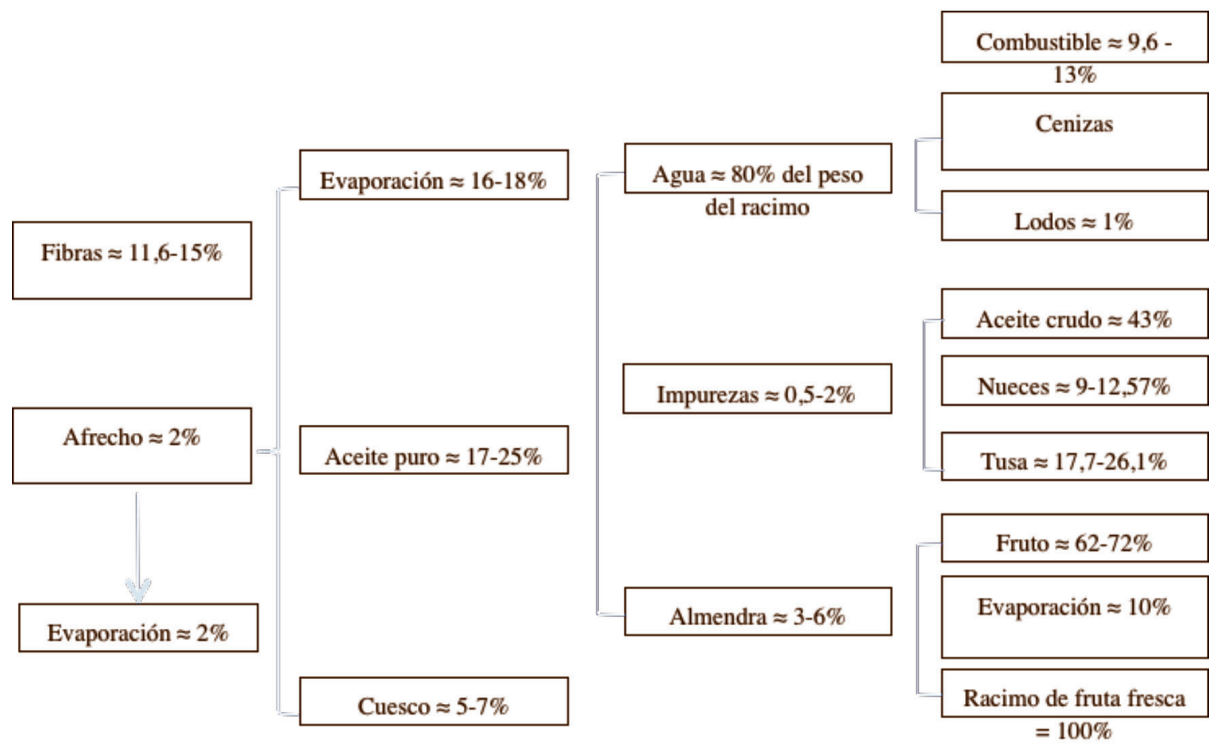

Fig. 1 Componentes del racimo de palma de aceite.

La composición de un racimo de palma aceitera se presenta en la Fig. 1. Luego de la recolección y almacenamiento del fruto, el racimo pasa a un proceso de esterilización, luego se descarta el fruto de su raquis para conducirlo a los digestores. El objeto del digestor es acondicionar el fruto, haciendo que se aparte el mesocarpio de las nueces para facilitar la extracción del aceite en el prensado.
Como se observa en la Fig. 2, los racimos de fruta fresca (1) son descargados (2) y acarreados hacia el área de esterilización (3). La esterilización se desarrolla con vapor a baja presión por espacio de 90 minutos. Los frutos se apartan de los racimos en la trilladora por un proceso mecánico (4). El raquis es traído hacia los camiones para ser usados en compostaje. 
La semilla es pasada hacia la digestión en donde con un proceso de rompimiento de las células se libra el aceite del fruto. El digestor usualmente colocado consiste de un envase cilíndrico caldeado con vapor ajustando con un agitador (5). El aceite se clarifica mediante una separación gravimétrica, la cual consiste en la diferencia de densidades (6). Se le retira humedad al aceite clarificado mediante un calentamiento en sistemas de tanques o al vacío. El agua que está contaminada con aceite como subproducto del proceso de clarificación se pasa a través de centrífugas con el fin de recuperar un poco de aceite.

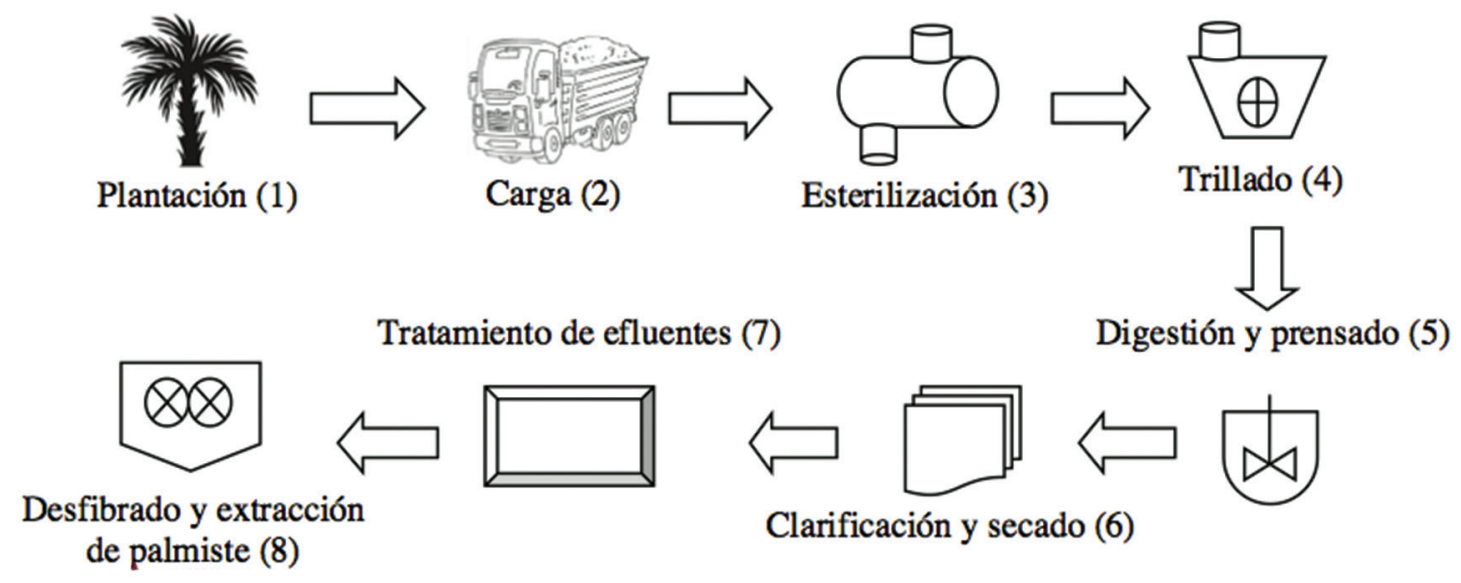

Fig. 2 Descripción del proceso tradicional de extracción del aceite de palma.

El residuo líquido se trata en el sistema de tratamiento de aguas residuales (7). Se apartan la mezcla formada de fibra y nueces. Se quebrantan las cáscaras de las nueces y se excluye el palmiste. El palmiste pasa a través del silo de secado y el aceite es prensado (8). El aceite se comercializa y la torta de palmiste se usa como forraje, la fibra y la cáscara son recogidas y se emplean como combustible en la caldera. Son precisamente los pasos (4) y (8) los de mayor interés en el presente trabajo por cuanto ese residuo agrario puede tener un valor agregado para la generación de energía con el fin de surtir la misma planta. El aceite extraído es recolectado donde le son separadas parte de las impurezas para el proceso como: fibras, arena, etcétera y luego es bombeada a la sección de clarificación.
Con la refinación del aceite crudo de palma se persigue la exclusión de sustancias inhibitorias de la producción de biodiesel. El proceso empieza con la adición de ácido cítrico y tierras de blanqueo que atrapan estos compuestos, a continuación mediante un filtrado son apartados del aceite. Después el aceite tratado se lleva hasta condiciones de vacío y alta temperatura, permitiendo con esto la evaporación y separación de los ácidos grasos libres obteniéndose el aceite refinado como producto final.

El proceso de producción de biodiesel se realiza por medio de una reacción química del aceite refinado (RBD: Refinado, blanqueado y desodorizado) con metanol en presencia de hidróxido de sodio como catalizador, formando una fase liviana de metilester (biodiesel) y una fase pesada de glicerina cruda (Fig. 3). 


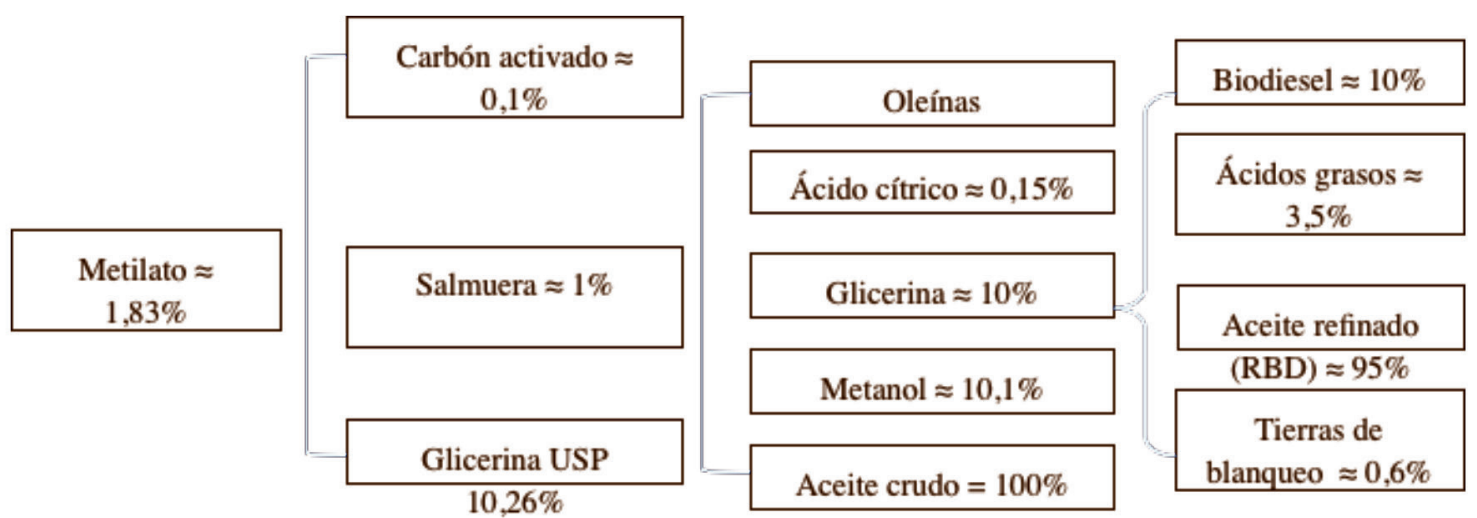

Fig. 3 Composiciones de los productos obtenidos en la fabricación de biodiesel a partir de aceite de palma.

El biodiesel es sometido a un lavado con agua caliente para su neutralización y posterior centrifugación con el fin de remover sales y otras impurezas. Este proceso es también conocido como de transesterificación y se produce también la glicerina como coproducto Fig. 4.

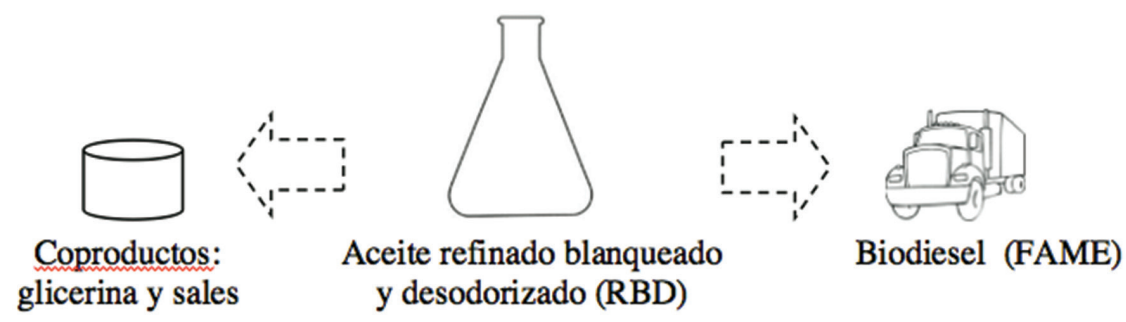

Fig. 4 Transesterificación del aceite

Proceso de purificación de la glicerina: La glicerina cruda derivada de la producción del biodiesel es llevada para la remoción de jabones mediante un proceso de acidificación, después se neutraliza formando una salmuera. Posteriormente, la glicerina es purificada a través de una destilación en una columna a condiciones de vacío y alta temperatura, pasa luego a filtración con carbón activado obteniéndose así la glicerina refinada.

\section{CONCEPTO DE BIOREFINERÍA EN UNA PLANTA EXTRACTORA DE ACEITE DE PALMA}

El aceite de palma es el aceite vegetal más producido en el mundo, en el caso de Colombia, se cultivó la palma africana (Elaeis guineensis) para consumo interno mediante su empleo en productos alimenticios tales como aceite de cocina, productos de panadería, margarina y manteca (Ortiz \& Fernández, 2000). En la década pasada se consideró al cultivo de palma como sustituto de cultivos ilícitos por lo que con el impulso gubernamental, además de expandirse el número de hectáreas sembradas, también se diversifico su producción. Entonces sus fracciones derivaron a usos no comestibles, entre los que se encuentra la fabricación de compuestos oleoquímicos tales como esteres metílicos, polioles, poliuretanos para jabónes, detergentes y compuestos de limpieza industrial y del hogar, aunque gran parte del aceite generado es exportado, así como el sobrante se procesa industrialmente en la fabricación de biodiesel. Sin embargo, la ley restringe las emisiones de particulado generados por la combustión de los combustibles derivados de biomasa, con normas restrictivas y difíciles de cumplir, ya que las tecnologías 
utilizadas en la mayoría de las plantas nacionales son antiguas, sumado a lo anterior, la reglamentación para los vertimientos contiene nuevos criterios para DBO, DQO, sólidos totales, grasas y aceites, compuestos fenólicos y herbicidas.

US\$ 450/ton aceite de palma crudo, es el costo de la extracción de palma nacional colombiana, mayor aún que el de otros países productores con menor producción como los países centroamericanos y Ecuador que ya alcanza iguales niveles de la producción nacional. La trasformación de las plantas extractoras a biorefinerías puede apoyar la competitividad de la industria palmera. Una estrategia consiste en implementar gradualmente módulos tecnológicos dentro de una clara estrategia para diversificar los subproductos así reducir el impacto ambiental y los costos de los procesos industriales actuales. La conversión de plantas procesadoras a biorefinerías cuenta con varias alternativas de uso de la biomasa de la palma, en la Fig. 5 se muestra las opciones de procesos. Una biorefinería es una estructura que integra procesos de conversión de biomasa y equipamiento para producir combustibles, energía y productos químicos a partir de la biomasa. El concepto de biorefinería es análogo al de refinerías de petróleo, los cuales producen productos y combustibles múltiples derivados del petróleo (García, 2013).

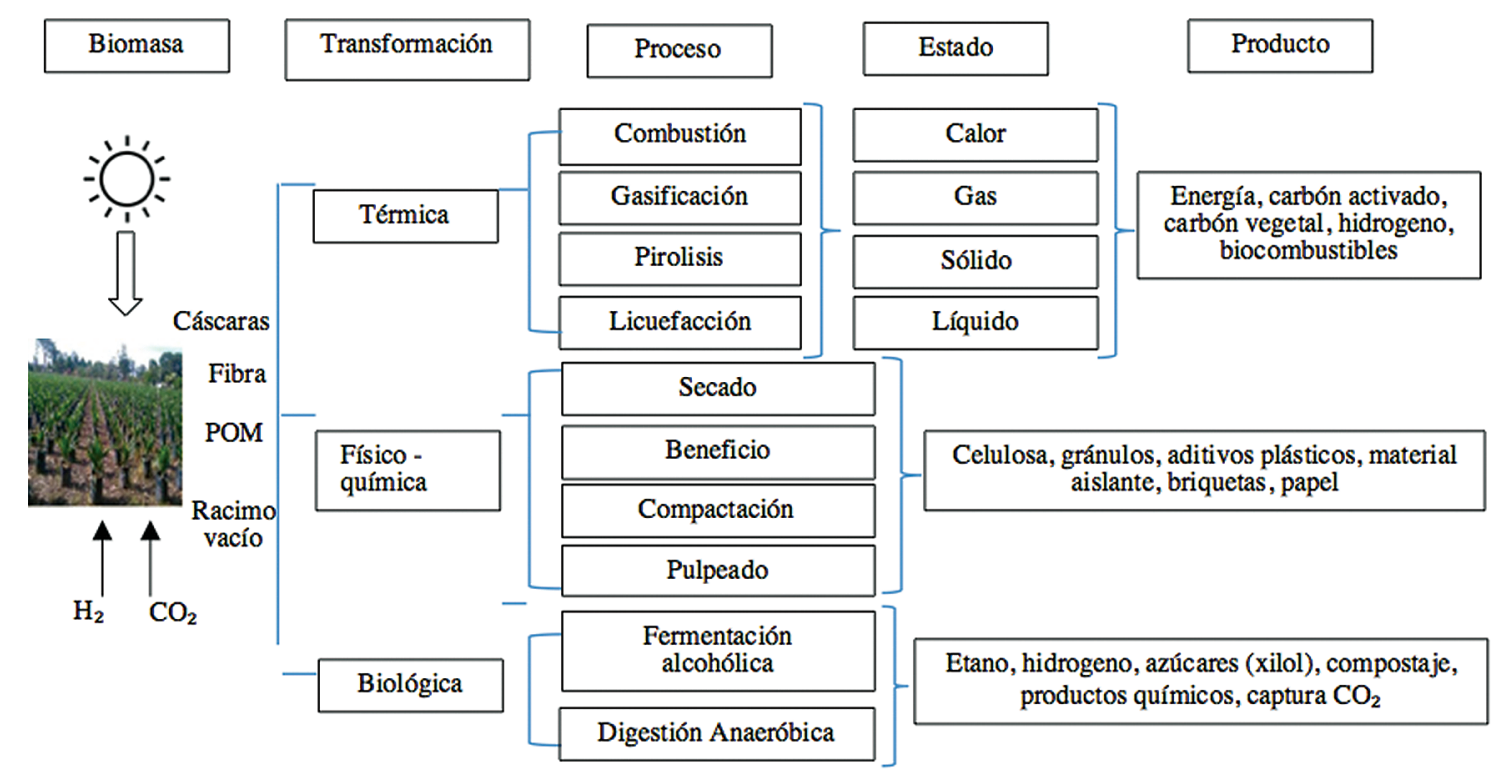

Fig. 5 Alternativas para el uso de la biomasa de la palma de aceite.

Tecnologías tradicionales se manejan para elaborar productos petroquímicos o bioproductos, según sea el caso (González-Delgado \& Kafarov, 2011; Mansoornejad et al. 2010; Pérez et al. 2017). La integración de las configuraciones de producción puede ampliarse en las biorefinerías para producir bienes con valor agregado (Bonomi, 2011; Moncada et al. 2015). Estos diseños generalmente aprovechan la máxima producción y provecho de una sola fuente de materia prima, y a veces no se tienen diferencias sobre la escasez de los insumos o de los impactos ambientales causados por su colecta o procesamiento. Una tecnología que contemple estas condiciones brindará un producto que además de proceder de una tecnología limpia, sería además innovadora al mercado. Una biorefinería representa una diversificación dentro de las agroindustrias existentes o el uso alternativo de la materia prima biológica a partir de las tecnologías que actualmente se usan con los recursos fósiles para obtener combustibles, productos químicos o la energía a partir de materiales derivados biológicamente 
(NREL, 2008). Si se acopian y trasladan de las plantas extractoras de aceite de palma (POM por su acrónimo en inglés) los otros materiales de desecho disponibles en el sector, en un mediano futuro la capacidad de estas biorefinerías podría extenderse (García, 2013; Wright \& Brown, 2007).

Una manera de aumentar el uso de la biomasa es mediante la cogeneración de energía lo cual contrasta con los modelos de negocio de las nuevas biorrefinerías concebidas para la utilización de desechos agrícolas. Si no se implementan sistemas de cogeneración y si las calderas existentes reducen el uso de la fibra debido a las limitaciones ambientales y solo consumen una fracción de lo que se utiliza actualmente para generar el vapor, habrá mayores cantidades de biomasa para la producción de combustibles y productos biológicos (Abdullah et al. 2015; Bridgwater, 2011; Chong, 2011; Rojas, 2011). El hecho de que la biomasa se encuentre en la planta de beneficio donde ya hay instalaciones y en la cual se obtiene biomasa todo el año, facilita la implementación de los conceptos de biorrefinería asociados con la producción del aceite de palma. Una sola planta procesadora con capacidad promedio (60 ton $\mathrm{RFF} / \mathrm{h}$ ) puede producir anualmente hasta 54.000 ton de racimos vacíos (tusas) (RV), 35.100 ton de fibra, 10.800 ton de cáscara y 141.750 ton de efluentes líquidos. La Figura 6 muestra un escenario de referencia general para una extractora común en Colombia (García, 2013).

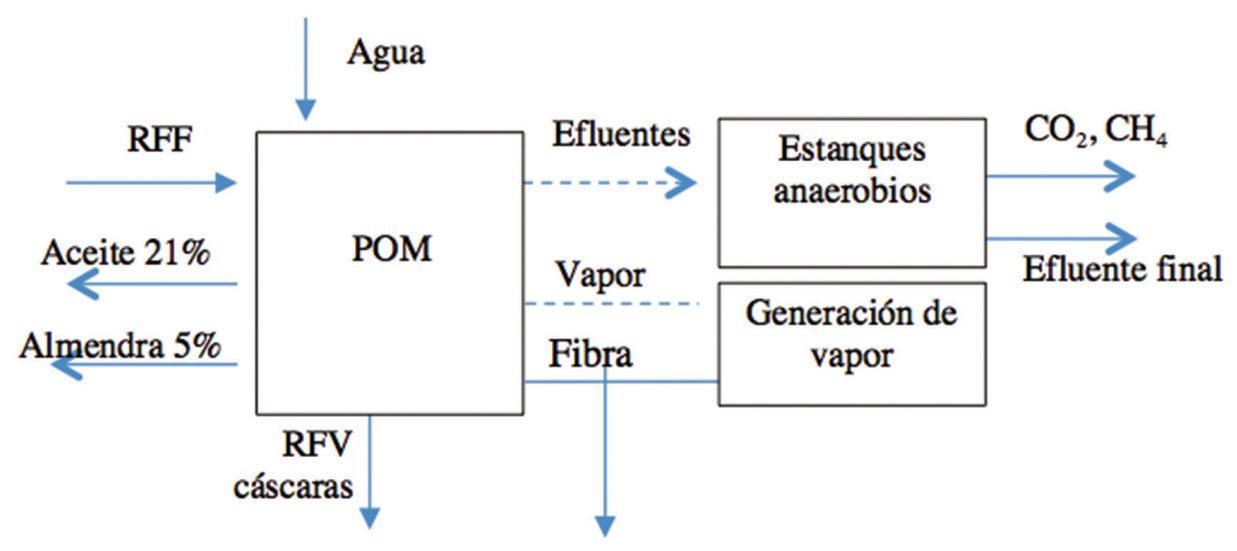

Fig. 6 Escenario general de las plantas extractoras en la que los productos principales son únicamente aceite de palma y almendra.

En la actualidad y con el Protocolo de Kioto para la captura y comercialización del $\mathrm{CO}_{2}$ mediante el Mecanismo de Desarrollo Limpio (MDL) y los compromisos adquiridos con la COP21, muchas POM han implementado proyectos de MDL a fin de participar en el mercadeo del $\mathrm{CO}_{2}$ (Conil, 2007; Tantitham et al. 2009), en Colombia se aprobó un proyecto MDL en el año 2007 (Fedepalma, 2007). Se han realizado varios estudios en los que la biomasa lignocelulósica en POM (González et al., 2008; Bakker, 2013; Carvajal, 2015; Castillo, 2011), se utiliza para producir enzimas de celulosa (Alam et al., 2009), biocompuestos (Shinoj et al., 2011), bioetanol (Goh \& Lee, 2010; Tan, Lee \& Mohamed, 2010; Yamada et al. 2010), hidrógeno y gas sintetizado (Chew \& Bhatia, 2008; Kelly-Yong et al.
2007), carbón activado (Guo \& Lua, 1998; Lua et al. 2006; Fan et al. 2011; Gómez et al. 2011) y compostaje (Baharuddin et al. 2010; Singh et al. 2010, Avendaño y Martínez, 2015). Se ha propuesto la integración de la producción del bioetanol a partir de materiales lignocelulósicos y la producción de biodiésel a partir del aceite de palma en una POM (Gutiérrez et al. 2009; Goh et al. 2010, Abdullah et al. 2016).

Otras opciones potenciales para las biorefinerías incluyen la producción de lípidos a partir de la biomasa y fermentar los azúcares derivados de los materiales lignocelulósicos (Loboguerrero, 2007; Hernández, 2015). Los azúcares se pueden obtener mediante rutas bioquímicas o termoquímicas. Esta es una iniciativa 
novedosa que han desarrollado investigadores en Washington State University (WSU) y puede utilizarse con los racimos vacíos como sustrato. Una POM posee la infraestructura para producir y comercializar aceites pero además con la integración de sus propios residuos ayudaría a incrementar la producción de energía (Unipalma, 2016).

En cuanto a la lignocelulosa, esta es una materia prima clave para la biorefinería. Para referirse a ella a menudo se utiliza el término "segunda generación", para significar que no sale de productos que a su vez sean alimento. Como se sabe, de la biomasa agrícola puede producirse energía en forma de: biodiésel (aceite residual/tortas prensadas), biogás, H2 y fermentación del etanol, biopolímeros, pirólisis de bioaceite y carbón vegetal, fibras de papel, paneles de construcción y materiales compuestos de disolución de celulosa, tablero aglomerante (lignina intrínseca), productos químicos "verdes" (adhesivos furfural), líquidos Fischer Tropsch a partir de biomasa.

Se calcula al alrededor de 50 millones de toneladas la biomasa de la palma de aceite, que en una escala global puede no verse como mucho, pero hay concentraciones en pocas áreas, y resulta interesante usarla para la biorefinería. Los productos de la biorefinería que se mencionan aquí son principalmente los polímeros carbohidratos que se convierten en azúcar, a partir de los cuales se pueden elaborar múltiples productos, como combustibles:

- Etanol. Es sobre el que se ha desarrollado mayor investigación.

- Butanol. Puede ser usado como remplazo del diésel y además es una plataforma para hacer diferentes productos químicos.

- Hidrógeno. Se puede producir de los carbohidratos, empleando bacterias.

El Análisis de Ciclo de Vida para el caso de biorefinerías derivadas de residuos de la palma aceitera se basa en investigaciones previas, sin embargo, no se ha reportado el impacto que podría generar por lo que este estudio permita identificar los posibles impactos ambientales $y$ áreas de mejora. El Análisis del Ciclo de Vida (ACV) también es una herramienta que permite comparar los efectos ambientales de las diferentes tecnologías (Bridgwater, 2011). En Colombia se desarrolló una ACV para determinar el efecto que tiene la producción de biodiésel y también sería una herramienta muy útil para estudiar el impacto ambiental de convertir las POM en biorrefinerías. La base de datos que alimentó esta ACV está disponible en Cenipalma para su uso posterior. (Yánez et al.2009) trabajaron en una aproximación a la ACV para la producción de biodiésel en Colombia y Brasil. Para el caso colombiano, como en la mayoría de los casos, se necesita información más precisa del asunto particular, por ejemplo, sobre agricultura tropical, suelos y productos específicos, entre otros, este trabajo se hizo durante meses, con expertos nacionales y en cooperación con Cenipalma y Cenicaña (Gauch, 2013).

\section{Alcance del estudio}

\section{A) Objetivo}

Desarrollar un Análisis de Ciclo de Vida para una biorefinería derivada de los residuos del cultivo de la palma aceitera.

\section{B) Función del sistema en estudio}

El sistema en estudio tiene como finalidad el aprovechamiento integral de los residuos en una extractora de aceite de palma con el objeto de incorporarlos a la cadena productiva mediante la integración de biocombustible y la cogeneración energética.

\section{C) Unidad funcional}

La unidad funcional es una medida del comportamiento de las salidas funcionales de un sistema y su propósito es proporcionar una referencia para las entradas y salidas del mismo. Esta referencia es necesaria para asegurar que la comparación de los sistemas se hace sobre una base común. Las unidades funcionales a estudiar son:

- $1 \mathrm{MJ}$ al punto de distribución.

- 1 vehículo-kilómetro.

- 1 ton de materias primas. 


\section{D) Límites de los sistemas}

El sistema incluye todos los procesos relacionados con el cultivo, el procesamiento, el transporte y el uso de biocombustibles y combustibles fósiles en Colombia. Asimismo la producción, mantenimiento, reutilización y disposición de la infraestructura, incluyendo construcciones y vías.

Los límites geográficos del sistema se definieron como de la puerta a la puerta ya que se tomó en cuenta el proceso de producción, es decir, desde que entran las materias primas al proceso, hasta que se obtiene el producto final de un caso genérico representativo de una extractora de aceite de palma colombiana. La información recopilada será para una producción del anual.

\section{E) Metodología de análisis}

La metodología de Análisis de Ciclo de Vida (ACV) es una herramienta de gestión ambiental de recopilación y evaluación de las entradas, resultados y los impactos ambientales potenciales de un sistema o producto durante su ciclo de vida. Esta metodología describe y analiza las corrientes que entran desde la naturaleza al sistema estudiado y las que salen del sistema a la naturaleza a lo largo del ciclo de vida, es decir, desde la extracción de materias primas y su agotamiento hasta emisiones al aire, agua y suelo así como los cambios de uso del terreno. La metodología seguida en este análisis de inventario, usa la aproximación conceptual del análisis de sistemas, en el sentido en que traza una frontera alrededor del sistema analizado y cuantifica las entradas y salidas a través de esa frontera. No siempre es posible tener todos los datos necesarios para la elaboración del inventario, por lo que es necesario, partir de una serie de suposiciones e hipótesis que permitan completar dichos datos. A continuación se detallan algunas de las principales hipótesis metodológicas que sustentan el desarrollo del inventario y facilitan su análisis (International Reference Life Cycle Data System (ILCD) Handbook, 2010).

Como se aprecia en la Fig. 7 se identificaron cada una de las etapas del proceso de producción y las materias primas utilizadas para realizar un diagrama de esquemático de entrada y salidas del sistema. Posteriormente, se calcularon las cantidades necesarias de cada materia prima para finalmente introducir los datos en el software SimaPro y calcular los impactos potenciales en cada etapa e identificar áreas de mejora.

\section{F) Requisitos de calidad de los datos}

Los datos sobre materias primas deben ser de la producción y las especificaciones de los equipos deben provenir de las operaciones que desarrollan el proceso (Das et al., 2007).

\section{1) Método de impacto}

El método de impacto utilizado fue TRACI 2.1 Versión 1.01, este es una herramienta que permite evaluar los impactos químicos y otros impactos ambientales, $\mathrm{y}$ fue desarrollado por la Agencia de Protección Ambiental de Estados Unidos EPA. 


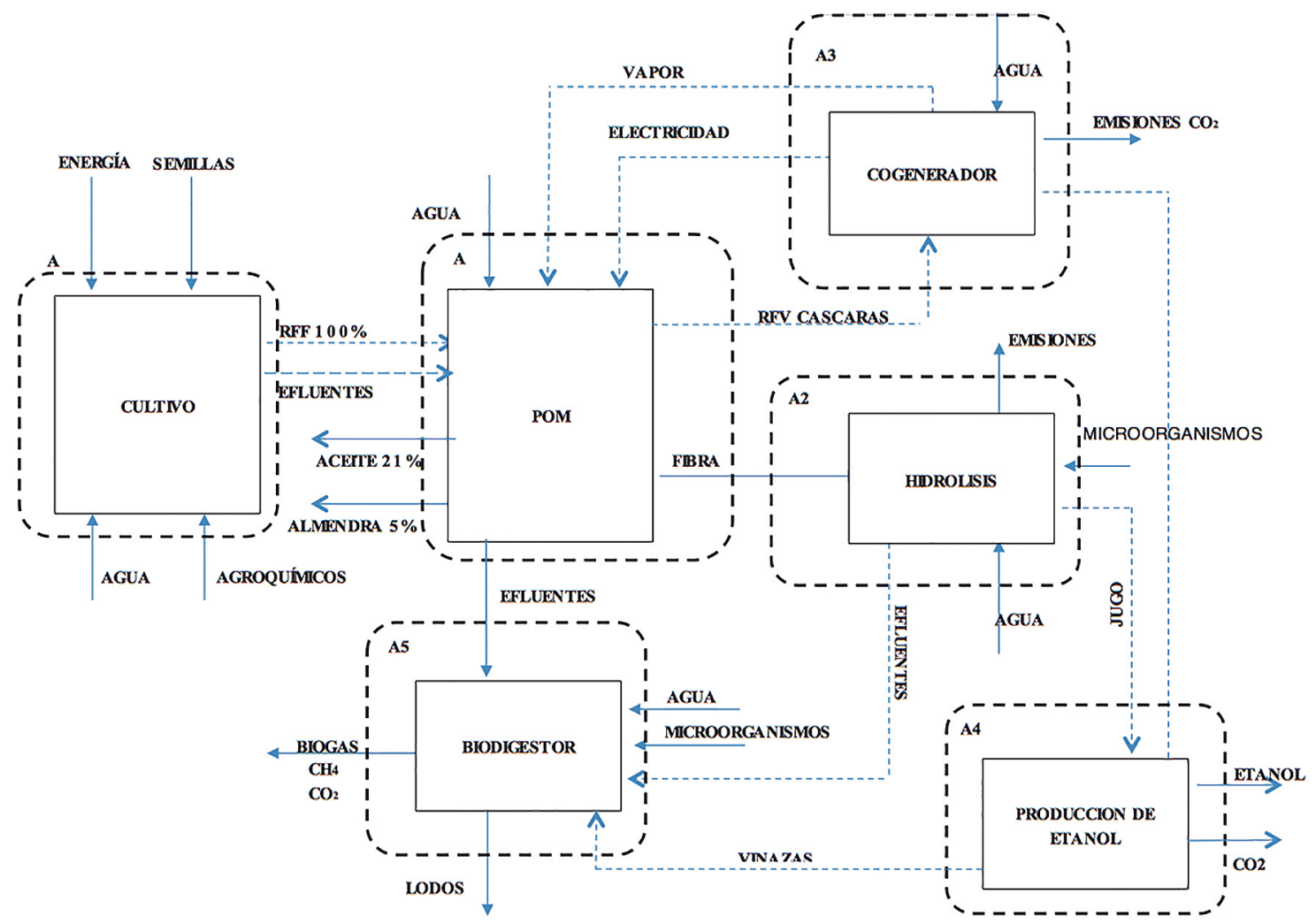

Fig. 7 Diagrama esquemático de entradas y salidas del sistema.

\section{2) Método de asignación:}

Se les asignó una parte del daño ambiental de la producción a todos los subproductos (por ejemplo torta de palmiste, compost, electricidad, etcétera) del biocombustible según su valor económico.

\section{G) Herramienta utilizada}

Para realizar el análisis de ciclo de vida se utilizó el software SimaPro 8 (C) Copyright PRÉ 2017. SimaPro es una marca registrada de PRÉ Consultants B.V. (Garrain, 2017b). El software cuenta con las siguientes características:

- Protocolos para la realización guiada de ACV'.

- Modificación en cualquier momento de todos los parámetros del ciclo de vida.

- Análisis tipo LCA (Life Cycle Assessment) y LCC (Life Cycle Cost)

- Redacción de informes de acuerdo con la normativa ISO 14040 e ISO 14044.
- Análisis de la incertidumbre de los datos, escenarios de fin de vida, análisis de sensibilidad y Monte Carlo.

- Exportar la información en formatos Ecospold y Excel.

\section{INVENTARIO PARA LAS ETAPAS Y PROCESOS CONSIDERADOS}

La recopilación de información primaria sobre el cultivo y el procesamiento de biocombustibles en el campo se extrae del estudio realizado por el Banco Interamericano de Desarrollo (BID, 2011), la información secundaria se toma de la base de datos Ecoinvent v2.2 (Database Ecoinvent "Palm oil, at oil mill, MY", 2017) y del Statical Yearbook (Fedepalma, 2015) y de otros autores especificados. 


\section{A) Etapa de cultivo.}

\section{TABla 1}

Entrada de fertilizantes minerales en kg por hectárea y año (4148 ha). (BID, 2011)

\begin{tabular}{|l|l|c|}
\hline \multicolumn{1}{|c|}{ Fertilizante mineral } & Unidades & Valor \\
\hline Urea & $\mathrm{kg} / \mathrm{ha} / \mathrm{año}$ & 111.72 \\
\hline Ammonium nitrate phosphate & $\mathrm{kg} / \mathrm{ha} / \mathrm{año}$ & 159.27 \\
\hline Potassio (chloride) & $\mathrm{kg} / \mathrm{ha} / \mathrm{año}$ & 322.75 \\
\hline Dolomitio & $\mathrm{kg} / \mathrm{ha} / \mathrm{año}$ & 228.74 \\
\hline Borax & $\mathrm{kg} / \mathrm{ha} / \mathrm{año}$ & 11.55 \\
\hline $\mathrm{N}$ & $\mathrm{kg} / \mathrm{ha} / \mathrm{año}$ & 64.77 \\
\hline P2O5 & $\mathrm{kg} / \mathrm{ha} / \mathrm{año}$ & 82.82 \\
\hline K2O & $\mathrm{kg} / \mathrm{ha} / \mathrm{año}$ & 193.65 \\
\hline $\mathrm{MgO}$ & $\mathrm{kg} / \mathrm{ha} / \mathrm{año}$ & 50.32 \\
\hline B2O3 & $\mathrm{kg} / \mathrm{ha} / \mathrm{año}$ & 5.54 \\
\hline
\end{tabular}

TABla 2

Composición de nutrientes en la tusa en base húmeda y seca.

\begin{tabular}{|l|c|c|c|c|}
\hline & $\mathbf{N}$ & $\mathbf{P 2 O 5}$ & K2O & MgO \\
\hline Peso seco & $0,54 \%$ & $0,14 \%$ & $2,77 \%$ & $0,32 \%$ \\
\hline Peso húmedo & $0,28 \%$ & 0,07 & $1,41 \%$ & $0,16 \%$ \\
\hline
\end{tabular}

TABLA 3

Agroquímicos aplicados en las diferentes áreas de cultivo por área de cultivo, kg/RFF/año. (BID, 2011)

\begin{tabular}{|l|c|c|}
\hline \multicolumn{1}{|c|}{ Agroquímico } & Unidades & \multicolumn{1}{c|}{ Valor } \\
\hline Glyphosate, at regional storehouse/RER S & $\mathrm{kg} / \mathrm{kg}$ RFF & $4.41 \mathrm{E}-05$ \\
\hline Bipyridylium-compounds, at regional storehouse/RER S & $\mathrm{kg} / \mathrm{kg}$ RFF & $1.888 \mathrm{E}-05$ \\
\hline Sulfony urea-compounds, at regional storehouse/RER S & $\mathrm{kg} / \mathrm{kg}$ RFF & $1.27 \mathrm{E}-05$ \\
\hline Ethoxylated alcohols (AE7), petrochemical, at regional plan/RER S & $\mathrm{kg} / \mathrm{kg}$ RFF & $1.57 \mathrm{E}-05$ \\
\hline Organophosphorus-compounds, at regional storehouse/RER S & $\mathrm{kg} / \mathrm{kg}$ RFF & $5.88 \mathrm{E}-05$ \\
\hline Acetamide-anilide-compounds, at regional storehouse/RER S & $\mathrm{kg} / \mathrm{kg}$ RFF & $6.08 \mathrm{E}-06$ \\
\hline Insecticides, at regional storehouse/RER S & $\mathrm{kg} / \mathrm{kg}$ RFF & $5.58 \mathrm{E}-06$ \\
\hline
\end{tabular}




\section{TABLA 4}

Consumo de combustible de las diferentes áreas de cultivo de palma en tonkm/kg RFF. (BID, 2011)

\begin{tabular}{|l|c|c|}
\hline \multicolumn{1}{|c|}{ Vehículo } & Unidades & \multicolumn{1}{c|}{ Valor } \\
\hline Transport, tractor and trailer & tonkm/kg & $9.1 \mathrm{E}-03$ \\
\hline Transport, lorry $>16 \mathrm{ft}$, fleet average & tonkm/kg & $9.4 \mathrm{E}-03$ \\
\hline Transport, passenger car, petrol, EURO3/person & tonkm/kg & $2.9 \mathrm{E}-04$ \\
\hline
\end{tabular}

TABLA 5

Emisiones a la atmósfera por la aplicación de fertilizantes. (BID, 2011)

\begin{tabular}{|l|c|c|}
\hline \multicolumn{1}{|c|}{$\begin{array}{c}\text { Emisiones } \\
\text { al aire }\end{array}$} & Unidades & Valor \\
\hline $\mathrm{NH} 3-\mathrm{N}$ & $\mathrm{kg} / \mathrm{kg} \mathrm{RFF}$ & $3.91 \mathrm{E}-04$ \\
\hline $\mathrm{N} 2 \mathrm{O}$ & $\mathrm{kg} / \mathrm{kg}$ RFF & $6.76 \mathrm{E}-04$ \\
\hline $\mathrm{NOx}$ & $\mathrm{kg} / \mathrm{kg}$ RFF & $1.42 \mathrm{E}-04$ \\
\hline
\end{tabular}

\section{TABLA 6}

Emisiones al suelo por la aplicación de pesticidas y fertilizantes. (BID, 2011)

\begin{tabular}{|l|c|c|}
\hline \multicolumn{1}{|c|}{ Emisiones } & Unidades & Valor \\
\hline $\mathrm{Cd}$ & $\mathrm{kg} / \mathrm{kg}$ RFF & $1.64 \mathrm{E}-07$ \\
\hline $\mathrm{Cu}$ & $\mathrm{kg} / \mathrm{kg}$ RFF & $-3.28 \mathrm{E}-06$ \\
\hline $\mathrm{Zn}$ & $\mathrm{kg} / \mathrm{kg} R F F$ & $-1.67 \mathrm{E}-05$ \\
\hline $\mathrm{Pd}$ & $\mathrm{kg} / \mathrm{kg} \mathrm{RFF}$ & $-6.94 \mathrm{E}-08$ \\
\hline $\mathrm{Ni}$ & $\mathrm{kg} / \mathrm{kg}$ RFF & $-7.47 \mathrm{E}-08$ \\
\hline $\mathrm{Cr}$ & $\mathrm{kg} / \mathrm{kg}$ RFF & $-7.47 \mathrm{E}-08$ \\
\hline $\mathrm{Hg}$ & $\mathrm{kg} / \mathrm{kg}$ RFF & $1.96 \mathrm{E}-07$ \\
\hline
\end{tabular}




\section{Tabla 8}

Emisiones al agua por el uso de fertilizantes. (BID, 2011)

\begin{tabular}{|c|c|c|}
\hline Emisiones & Unidades & Valor \\
\hline \multicolumn{3}{|l|}{ Nitrate } \\
\hline $\mathrm{P}$ & $\mathrm{mm}$ & 2500 \\
\hline$S$ & $\mathrm{~kg} \mathrm{~N} / \mathrm{ha} / \mathrm{año}$ & 64.77 \\
\hline $\mathrm{C}$ & día/contenido & 4.9 \\
\hline $\mathrm{L}$ & Root depth (m) & 1 \\
\hline C org & $\%$ & 0.81 \\
\hline Bulk density & ton oil $/ \mathrm{m} 3$ & 1.4 \\
\hline Corg EMPA & ton $\mathrm{C} / 3000 \mathrm{~m} 3$ & 34 \\
\hline $\mathrm{N}$ org & $\mathrm{kg} / \mathrm{ha}$ & 6.05 \\
\hline $\mathrm{U}$ & $\mathrm{kg} \mathrm{N} /$ ton yield & 39.46 \\
\hline $\mathrm{N}$ & kg NO3/kg plant & $3.6 \mathrm{E}-03$ \\
\hline \multicolumn{3}{|c|}{ Phosphorous to surface water } \\
\hline A & Potential long term manual soil loss ton/ha/year & 2.24 \\
\hline $\mathrm{R}$ & Erositivy factor $\mathrm{MJ} \mathrm{mm} / \mathrm{ha} / \mathrm{h} / \mathrm{anno}$ & 2322.66 \\
\hline $\mathrm{k}$ & Erodibility factor ton $\mathrm{h} / \mathrm{MJ} \mathrm{mm}$ & 0.13 \\
\hline LS & Slope factor & 0.3 \\
\hline$S$ & Slope \% & 0.002 \\
\hline $\mathrm{L}$ & Lenght $\mathrm{mm}$ & 100 \\
\hline $\mathrm{C} 1$ & Crop factor & 0.2 \\
\hline $\mathrm{C} 2$ & Tillage factor & 0.25 \\
\hline $\mathrm{P}$ & Practice factor & 0.5 \\
\hline $\mathrm{Pe}$ & $\mathrm{Kg} \mathrm{P} / \mathrm{kg}$ cane & $4.55 \mathrm{E}-05$ \\
\hline \multicolumn{3}{|c|}{ Phosphate to surface water: run-off and drainage } \\
\hline $\mathrm{kr}$ & Average run-off $\mathrm{kgP} / \mathrm{ha}$ año & 0.18 \\
\hline $\mathrm{f}$ & Mineral fertilizer kg P/ha & 82.82 \\
\hline $\mathrm{Cm}$ & $\mathrm{Kg} \mathrm{P} / \mathrm{kg}$ cane & $3.28 \mathrm{E}-05$ \\
\hline
\end{tabular}




\section{B) Etapa de extracción de aceite}

TABla 9

Entrada de materia y energía

\begin{tabular}{|l|c|c|}
\hline \multicolumn{1}{|c|}{ Entrada } & Unidad & Escenario promedio \\
\hline Racimos de fruto fresco (RFF) & ton & 100 \\
\hline Agua & ton & 109.84 \\
\hline Autogeneración de electricidad & $\mathrm{kWh}$ & 740.12 \\
\hline Electricidad de la red & $\mathrm{kWh}$ & 1358.11 \\
\hline Electricidad diesel & $\mathrm{kWh}$ & 19.08 \\
\hline Vapor & $\mathrm{m} 3$ & 43.35 \\
\hline
\end{tabular}

TABla 10

Productos, subproductos y residuos

\begin{tabular}{|l|c|c|}
\hline \multicolumn{1}{|c|}{ Salida } & Unidad & Escenario promedio \\
\hline Aceite crudo de palma & ton & 21.38 \\
\hline Tusa & ton & 21.34 \\
\hline Aceite de palmiste & ton & 2 \\
\hline Harina de palmiste & ton & 2.86 \\
\hline Agua residual & ton & 97.17 \\
\hline Fibra & ton & 13.16 \\
\hline Cáscaras de nueces & ton & 7.9 \\
\hline
\end{tabular}

Tabla 11

Producción de energía

\begin{tabular}{|l|c|c|c|c|}
\hline \multicolumn{1}{|c|}{ Parámetro } & Unidad & RFF & Cáscara & Fibra \\
\hline Poder calorífico inferior & $\mathrm{MJ} / \mathrm{kg}$ & 6.03 & 12.57 & 8.98 \\
\hline Humedad & $\%$ & 24.94 & 6.16 & 28.76 \\
\hline C & $\%$ & 54.3 & 51.8 & 58.9 \\
\hline H & $\%$ & 18.7 & 25.1 & 20.15 \\
\hline S & $\%$ & 0.22 & 0.3 & 0.24 \\
\hline N & $\%$ & 3.8 & 5.15 & 4.21 \\
\hline O & $\%$ & 11.02 & 12.35 & 8.62 \\
\hline Contenido de ceniza & $\%$ & 8.93 & 4.96 & 5.55 \\
\hline
\end{tabular}


Análisis de ciclo de vida para una biorefinería derivada de residuos agrícolas de palma aceitera (Elaeis guineensis)

Tabla 12

Infraestructura y maquinaria del proceso de extracción

\begin{tabular}{|l|c|c|c|}
\hline \multicolumn{1}{|c|}{ Proceso } & Cantidad & Unidad & Nombre ecoinvent \\
\hline $\begin{array}{l}\text { Extracción } \\
\text { de aceite }\end{array}$ & $1.00 \mathrm{E}-04$ & piece & Oil mill/CH \\
\hline \multirow{2}{*}{ Caldera } & $8.67 \mathrm{E}-05$ & piece & Cogen unit 6400kWth, Wood burning, buliding/CH \\
\cline { 2 - 4 } & $3.47 \mathrm{E}-04$ & piece & Cogen unit 6400kWth, Wood burning, common components for \\
heat+electricity/CH
\end{tabular}

TABLA 13

Rendimientos del procesamiento del fruto de la palma de aceite (Avendaño y Martínez, 2015)

\begin{tabular}{|l|c|c|}
\hline \multicolumn{1}{|c|}{ Producto } & Kg por ton de RFF & \% \\
\hline Aceite crudo & 207.7 & 15.37 \\
\hline Fibra & 151.1 & 11.18 \\
\hline Racimo vacío & 231.5 & 6.29 \\
\hline Cáscaras & 85 & 3.14 \\
\hline Nuez (almendra) & 42.2 & 45.46 \\
\hline Efluentes líquidos* & 614.1 & 1.43 \\
\hline Aceite perdido & 19.3 & \\
\hline
\end{tabular}

* Se agregan $350,9 \mathrm{~kg}$ de agua de proceso.

\section{TABla 14}

Poder calorífico de residuos sólidos de una extractora de aceite de palma (Duarte, 2006)

\begin{tabular}{|l|c|}
\hline \multicolumn{1}{|c|}{ Residuo } & Energía (kcal/kg) \\
\hline Fibra & 4420 (base seca) \\
\hline Cáscara & 4950 (base seca) \\
\hline Racimo vacío & 3370 \\
\hline
\end{tabular}


Revista Especializada en Ingeniería

TABLA 15

Consumo de recursos y energía en procesos de extracción de aceite de palma (UIS-IDEAM, 1999)

\begin{tabular}{|l|c|c|c|}
\hline \multicolumn{1}{r|}{ Recurso } & \multicolumn{2}{c|}{ Consumo por ton de } \\
\hline Agua & Unidades & RFF & Aceite crudo \\
\hline Fibra (Combustible) & ton & 1.2 & 5.78 \\
\hline ACPM (Combustible) & ton & 0.15 & 0.72 \\
\hline Aire & Gal & 1 & 4.81 \\
\hline Energía & M3 & 5300 & 25517 \\
\hline
\end{tabular}

\section{C) Etapa de hidrolisis}

TABla 16

Escala de producción (racimos de fruta vacíos)

\begin{tabular}{|l|c|c|c|c|}
\hline \multicolumn{1}{|c|}{ Tamańo de la planta } & Tipo & RFF/d & RFF/día (w) & RFF/año (dw) \\
\hline $30 \mathrm{~T} / \mathrm{H}$ & Mediana & $700 \mathrm{~T}$ & 210 & $26.9 \mathrm{KT}$ \\
\hline $90 \mathrm{~T} / \mathrm{H}$ & Grande & $2100 \mathrm{~T}$ & 630 & $80.6 \mathrm{KT}$ \\
\hline $150 \mathrm{~T} / \mathrm{H}$ & Muy grande & $3500 \mathrm{~T}$ & 1050 & $134 \mathrm{KT}$ \\
\hline
\end{tabular}

TABLa 17

Entrada para la producción de $100 \mathrm{~kg}$ de racimo de fruto vacíos (Bakker, 2013)

\begin{tabular}{|l|c|c|c|}
\hline \multicolumn{1}{|c|}{ Materia } & Composición (\%) & Cantidad & Unidad \\
\hline Glucano & 37 & 37 & $\mathrm{~kg}$ \\
\hline Xilano & 24 & 24 & $\mathrm{~kg}$ \\
\hline Arabinano & 2.4 & 2.4 & $\mathrm{~kg}$ \\
\hline Lignina & 24 & 24 & $\mathrm{~kg}$ \\
\hline Ceniza & 5 & 5 & $\mathrm{~mm}$ \\
\hline Tamaño de partícula & & $>25$ & $\mathrm{Kg} / \mathrm{m} 3$ \\
\hline Densidad aparente & & $80-100$ & \\
\hline Contenido de humedad & $40-60$ & & \\
\hline
\end{tabular}




\section{D) Etapa biodigestor}

\section{TABLA 18}

Vertimientos de los efluentes de una extractora de aceite de palma. (Duarte, 2006)

\begin{tabular}{|l|c|}
\hline \multicolumn{1}{|c|}{ Origen } & m m $^{3} /$ ton RFF \\
\hline Aguas lodosas de clarificación & 0.55 \\
\hline Condensados de esterilización & 0.10 \\
\hline Aguas de palmistería & 0.05 \\
\hline Aguas de lavado (limpieza de pisos y purgas) & 45 \\
\hline Efluente total sin fugas, ni purgas de desarenador y sedimentador & 0.80 \\
\hline
\end{tabular}

\section{TABLA 19}

Indicadores energéticos y de producción de biogás en la agroindustria de palma

\begin{tabular}{|l|c|c|}
\hline \multicolumn{1}{|c|}{ Indicador } & Unidades & Valor \\
\hline Producción de biogás & $\mathrm{m}^{3} / \mathrm{ton} \mathrm{RFF}$ & 182 \\
\hline Producción de biogás (lagunas) & $\mathrm{m}^{3} / \mathrm{kg}$ DQO removido & $0.19-0.25$ \\
\hline Poder de generación de energía eléctrica (EE) & $\mathrm{kwh} / \mathrm{m}^{3}$ & 1.8 \\
\hline Demanda de EE de una extractora de aceite de palma & $\mathrm{kwh} /$ ton RFF & $16-20$ \\
\hline EE producido por biogás generado & $\mathrm{kwh} /$ ton RFF & 32.76 \\
\hline
\end{tabular}

TABLA 20

Composición aproximada de biogás (InCOnTEC-Aene, 2003; Duarte, 2006)

\begin{tabular}{|l|c|c|}
\hline \multicolumn{1}{|c|}{ Compuesto } & Formula & \% volumen \\
\hline Metano & $\mathrm{CH}_{4}$ & $60-80$ \\
\hline Dióxido de Carbono & $\mathrm{CO}_{2}$ & $30-40$ \\
\hline Hidrógeno & $\mathrm{H}_{2}$ & $0-1$ \\
\hline Nitrógeno & $\mathrm{N}_{2}$ & $1-2$ \\
\hline Monóxido de Carbono & $\mathrm{CO}$ & $0-1,5$ \\
\hline Oxígeno & $\mathrm{O}_{2}$ & 0,1 \\
\hline Sulfuro de Hidrógeno & $\mathrm{H}_{2} \mathrm{~S}$ & $0-1$ \\
\hline Vapor de agua & $\mathrm{H}_{2} \mathrm{O}$ & 0,3 \\
\hline
\end{tabular}


TABLA 21

Caracterización de efluentes de palmas extractoras en Colombia (Avendaño \& Martínez, 2015)

\begin{tabular}{|l|c|c|c|}
\hline \multicolumn{1}{|c|}{ Parámetro } & Unidades & Intervalo & Promedio \\
\hline $\mathrm{pH}$ & - & $3.87-5.25$ & 4.55 \\
\hline Temperatura & ${ }^{\circ} \mathrm{C}$ & $53-77$ & 67.4 \\
\hline DBO5 & $\mathrm{mg} / \mathrm{l}$ & $18700-175521$ & 48873 \\
\hline DQO & $\mathrm{mg} / \mathrm{l}$ & $45256-232000$ & 79730 \\
\hline Sólidos totales & $\mathrm{mg} / \mathrm{l}$ & $32482-111029$ & 45670 \\
\hline Sólidos suspendidos & $\mathrm{mg} / \mathrm{l}$ & $19129-88258$ & 35105 \\
\hline Sólidos sedimentables & $\mathrm{mg} / \mathrm{l}$ & $125-1000$ & 677 \\
\hline Sólidos totales volátiles & $\mathrm{mg} / \mathrm{l}$ & $26530-98445$ & 48988 \\
\hline $\mathrm{P}$ & $\mathrm{mg} / \mathrm{l}$ & $15.7-113.6$ & 66.1 \\
\hline $\mathrm{N}$ & $\mathrm{mg} / \mathrm{l}$ & $67.5-695$ & 284.1 \\
\hline Grasas y aceites & $\mathrm{mg} / 1$ & $6480-80701$ & 18747 \\
\hline Acidez total & $\mathrm{mg} / 1$ & $750-20548$ & 1611 \\
\hline
\end{tabular}

TABLA 22

Características del biogás (Duarte, 2006)

\begin{tabular}{|c|c|c|}
\hline Propiedad & Unidades & Valor \\
\hline \multicolumn{3}{|l|}{ Planta extractora de aceite de palma } \\
\hline Capacidad de extracción & Ton RFF/día & 80 \\
\hline Tiempo de operación diario & horas & 8 \\
\hline Efluente producido & $\mathrm{m}^{3} / \mathrm{día}$ & 65 \\
\hline Efluente producido por ton de RFF & $\mathrm{m}^{3} /$ ton RFF & 0.8 \\
\hline \multicolumn{3}{|l|}{ Biodigestor } \\
\hline Capacidad de tratamiento & $\mathrm{m}^{3} / \mathrm{día}$ & 65 \\
\hline Tiempo de retención & días & 8 \\
\hline Producción de biogás & $\mathrm{m}^{3} / \mathrm{día}$ & 800 \\
\hline $\mathrm{CH}_{4}$ & $\%$ volumen & 65 \\
\hline $\mathrm{CO}_{2}$ & $\%$ volumen & 34 \\
\hline $\mathrm{H}_{2} \mathrm{~S}$ & $\%$ volumen & 0.18 \\
\hline Biogás retenido por DQO removido & $\mathrm{m} 3 /$ ton RFF & 0.55 \\
\hline Biogás obtenido por ton RFF & $\mathrm{m}^{3} /$ ton RFF & 10 \\
\hline Biogás obtenido por $\mathrm{m} 3$ de efluente & $\mathrm{m}^{3} / \mathrm{m}^{3}$ & 12 \\
\hline \multicolumn{3}{|l|}{ Sistema de generación eléctrica } \\
\hline EE requerida para el proceso & kwh & 200 \\
\hline Consumo total ACPM (sin biogás) & Gal/día & 80 \\
\hline Consumo de ACPM & $\%$ & 50 \\
\hline Ahorro anual de ACPM (Aprox.) & Gal/año & 10600 \\
\hline Consumo de biogás & $\mathrm{m}^{3} / \mathrm{dí} a$ & 320 \\
\hline EE generada por $\mathrm{m} 3$ de biogás & $\mathrm{kwh} /$ ton RFF & 2.5 \\
\hline EE generada por ton RFF & $\mathrm{kwh} /$ ton RFF & 25 \\
\hline Excedente de biogás & $\mathrm{m}^{3} / \mathrm{día}$ & 480 \\
\hline
\end{tabular}




\section{Evaluación DEL IMPACTO DEL CICLO DE VIDA}

\section{A) Interpretación}

La fase de interpretación en un estudio de ACV busca a través del análisis de los resultados tanto de la fase de Inventario y como de la Evaluación de Impacto del Ciclo de Vida, simplificar y dilucidar los aspectos notables que permitan establecer conclusiones conformes con la definición del objetivo y alcance planteados. La interpretación de los resultados en un ACV busca la caracterización de las variables que afecta el resultado final y sus interconexiones. Durante esta fase se identifican etapas que presentan una contribución importante, no sólo en términos de cantidad, sino también, por el porcentaje de participación. El propósito es identificar las principales cargas ambientales y por ende los aspectos del sistema evaluado que puedan o deban mejorarse.

En los siguientes apartados se describe el análisis de sensibilidad que se ha desarrollado en este estudio de $\mathrm{ACV}$, como parte de la fase de interpretación después de realizar un análisis del inventario para identificar áreas de mejora dentro del proceso (Garrain, 2017a, Naranjo, 2017).

\section{B) Análisis de inventario de Ciclo de Vida}

El análisis de inventario es un proceso de cuantificación de los flujos de energía y materiales que entran y salen de una actividad durante su ciclo de vida. Un análisis del inventario es fundamentalmente un balance de materia y energía del sistema, aunque también puede incluir otros parámetros como: utilización de suelo, radiaciones, ruido, vibraciones, biodiversidad afectada, entre otros aspectos (Delivan \& Gnansunou, 2013).

Los aspectos a tener en cuenta en la realización de un análisis de inventario de ciclo de vida, son aquellos que relacionan los flujos de materiales y energía con la unidad funcional, la necesidad de asignación y los métodos de asignación disponibles, y la disponibilidad de los datos. Estos aspectos se mencionan a continuación:
- Descripción de los procesos unitarios (balance de materia y energía)

- Procedimientos de cálculo (deben estar referidos a la unidad funcional)

- Criterios de asignación

- Asignación de flujos a productos y co-productos según lo establecido en la definición de objetivo y alcance

- Procedimientos de recopilación de datos

- Realización de cálculos

La metodología de la Evaluación del Impacto del Ciclo de Vida para determinar los siguientes impactos, los cuales se presentarán en una segunda entrega para:

- GEI calentamiento global gwp [kg_CO $\mathrm{CO}_{2}$-eq.]

- Acidificación acid [kg SO $\mathrm{k}_{2}$-eq]

- Eutrofización eutro [kg $\mathrm{PO}_{4}$-eq.]

- Ecotoxicidad etox [pt.]

\section{ConClusiones}

El alcance de la investigación en curso es evaluar el ACV en el cultivo de palma africana colombiana, con el objeto de inventariar los suministros de la agricultura y el consumo en una muestra representativa del cultivo de palma. Igualmente, recopilar información en este ámbito para la futura toma de decisiones hacia la sostenibilidad de los recursos y la optimización de los procesos por parte de las empresas de cultivadores de palma aceitera como parte de su política de responsabilidad ambiental (Torres e Israel, 2017).

Actualmente la idea de una economía circular tiene un papel protagónico en las agendas comerciales mundiales al punto de desvincularse el crecimiento económico de las limitaciones de recursos. La definición de 'economía circular' no se encuentra disponible como un solo concepto, pero está claro que, a diferencia del enfoque tradicional de toma-toma-consume-elimina, busca maximizar el valor en cada punto de la vida de un producto (Muñoz y Herrera, 2017).

Esta revisión tiene como propósito presentar un desarrollo metodológico inicial, así como las aplicaciones 
de enfoques de ciclo de vida independientes o integrados: huella ambiental - evaluación de la sostenibilidad del ciclo de vida para respaldar la evaluación de estrategias de economía circular en un escenario 'cradle to cradle' de la cuna a la cuna, en una segunda entrega se presentará un análisis detallado de los resultados procesados mediante el software Simapro.

\section{Agradecimiento}

Un reconocimiento especial para la Fundación Carolina por su colaboración mediante el Programa de Becas para Estancias Postdoctorales, igualmente al apoyo brindado por el equipo humano del Departamento de Energía del Centro de Investigaciones Energéticas, Medioambientales y Tecnológicas - CIEMAT (Madrid, España).

\section{REFERENCIAS}

Abdullah S., Shirai Y., Mohd A., Bahrin E., Hassan M 2015, Fresh oil palm frond juice as renewable, non-food, non-cellulosic and complete medium for direct bioethanol production. Industrial Crops and Products, 63, 357-361.

Abdullah S., Shirai Y., Mohd A., Mustapha M., Hassan M 2016, Case study: Preliminary assessment of integrated palm biomass biorefinery for bioethanol production utilizing non-food sugars from oil palm frond petiole. Energy Conversion and Management, 108, 233-242.

Alam, M. Z., Mamun, A., Qudsieh, I.Y., Muyibi, S.A., Salleh, H.M. \& Omar, N.M 2009, Solid state bioconversion of oil palm empty fruit bunches for cellulase enzyme production using a rotary drum bioreactor. Biochemical Engineering Journal, 46(1), 61-64.

Arévalo D 2012, Una mirada a la agricultura de Colombia desde su huella hídrica. WWF Colombia. Recuperado el 04 de Noviembre de 2014 en: http://www.huellahidrica.org/Reports/ Arevalo-2012-HuellaHidricaColombia.pdf.

Arrieta, R., Teixeira F., Yañez E., Lora E., Castillo E 2007, Cogeneration potential in the Colombian palm oil industry: Three case studies. Biomass and Bioenergy, 31(7), 503-511.

Asociación española para la calidad 2014, La Huella Hídrica. Centro Nacional de Información de la calidad. Recuperado el 04 de Noviembre de 2014 en: http://www.aec.es/c/document_library/get_file?uuid=935d5e6d-b631-44ad-a6e3c8861d0813d 2\&groupId=10128.
Avendaño F., Martínez J 2015, "Recuperación de lodos de las lagunas de oxidación provenientes del proceso de extracción de aceite de palma, para usar como abono en cultivos de palma africana". Tesis. Universidad Nacional Abierta y a Distancia. 63p.

Baharuddin S., Hock L., Yusof, Z., Abdul, N., Shah, U., Hassan A., Wakisaka M 2010, Effects of palm oil mill effluent (pome) anaerobic sludge from $500 \mathrm{~m} 3$ of closed anaerobic methane digested tank on pressed-shredded empty fruit bunch (RV) composting process. African Journal of Biotechnology, 9(16), $2427-2436$

Barón J 2015, "Evaluación de la huella hídrica de C.I. Flores La Conejera”. Tesis. Universidad de La Salle, Bogotá, Colombia.

Bakker R. 2013, Estado e implicaciones de los residuos de la biomasa de palma de aceite. Palmas. 34, Tomo II, 111-119.

Banco Interamericano de Desarrollo (BID) 2011, Biocombustibles Colombia. Consorcio CUE - Proyecto Estrategias de energía sostenible y biocombustibles para Colombia ATN/JC-10826CO y ATN/JF-10827-CO - Evaluación del Ciclo de Vida de la cadena de producción de biocombustibles en Colombia. Medellín, Colombia.

Basiron Y., Darus A 1997, La industria de la palma de aceite de contaminación a cero desechos. Palmas, 18, 1, 51-65.

Baumann H, Tillman A.M 2004, The Hitch Hiker's Guide to LCA. Ed. Studentlitteratur AB. Lund, Sweeden.

Bello D., Castellanos F 2016, "Propuesta de módulos de riego para la producción agrícola de caña panelera RD7511 en la finca La Dulzura del municipio de Villeta Cundinamarca”. Tesis. Universidad de La Salle, Bogotá, Colombia.

Bonomi, A 2011, "Virtual Sugarcane Biorefinery: A tool to compare the sustainability of different technological alternatives". PASI 2011 - Process Modeling and Optimization for Energy and Sustainability. Angrados Reis, RJ, Brazil. Retrieved from http://cepac.cheme.cmu.edu/pasi2011/library/bonomi/Presentation_VSB_Bonomi.pdf

Bridgwater, T 2011, "A methodology for the generation and evaluation of biorefinery chains". IEA Bioenergy Agreement Task 34 Newsletter - PyNe 29, pp. 18-19. Retrieved from http:// www.pyne.co.uk/Resources/user/PyNe Issue 29 v1.pdf

Cabrera A., Camelo I 2015, "Construcción y puesta en marcha de una planta piloto de procesos avanzados PTAP con fines pedagógicos en el Programa de Ingeniería Ambiental y Sanitaria”. Tesis. Universidad de La Sal e, Bogotá.

Carrero D., Loza M 2016, "Evaluación del uso y gestión del recurso hídrico en el cultivo de plátano de la Finca Villa Mariana en Puente Nacional Santander". Tesis. Universidad de La Salle, Bogotá.

Carvajal J 2015, "Evaluación técnica, económica y ambiental de una biorefinería a partir de materias primas con alto contenido en lignina en el contexto colombiano". Tesis. Universidad Nacional de Colombia. Manizales. 188p. 
Castañeda J., Ramírez J 2016, "Evaluación del uso gestión del recurso hídrico para el cultivo de cebolla de bulbo en la Finca Buena Vista de la vereda Flores" - Cundinamarca. Tesis. Universidad de La Salle, Bogotá.

Castillo, E 2016, Producción de etanol celulósico a partir de las tusas de palma: perspectiva de Ecopetrol. Palmas, 37(Especial Tomo II), pp. 115-118.

Chew T., Bhatia, S 2008, Catalytic processes towards the production of biofuels in a palm oil and oil palm biomass-based biorefinery. Bioresource Technology, 99(17), 7911-7922.

Chong, K. J 2011, A methodology for the generation and evaluation of biorefinery process chains, in order to identify the most promising biorefineries for the EU. Aston University.

Conil, P 2007, Biogas and C.E.R. Generation in Palm Oil Mills in Latin America. Proceedings of the PIPOC 2007 International Palm Oil Congress (Chemestry \& Technology) (pp. 262 - 263). Kuala Lumpur.

Das, K., García J., García M 2007, Overview of a Biorefinery and opportunities in the palm oil sector. Palmas, 28 (Especial), 91100.

Database Ecoinvent "Palm oil, at oil mill, MY" 2017.

Deaza D., Godoy A 2016, "Evaluación del uso y gestión del recurso hídrico en la Finca San Francisco de la vereda Guadalemo para la producción de maíz en el municipio del Espinal - Tolima”. Tesis. Universidad de La Salle, Bogotá.

Delivan M., Gnansounou E 2013, Life cycle enviromental impacts of a prospective palm-based biorefinery in Pará State-Brazil. Bioresource Technology. 150, 438-446.

Duarte S 2006, "Estudio de factibilidad tecnoeconómica y ambiental para el aprovechamiento del biogás producido en el tratamiento de efluentes de las plantas extractoras de aceite de palma de la zona de Puerto Wilches”. Tesis. Universidad Industrial de Santander. Bucaramanga.

Escobar M., Novoa M 2016, "Eficiencia del uso de microorganismos para el tratamiento de aguas residuales domésticas en una planta de tratamiento de agua residual”. Tesis. Universidad de La Salle, Bogotá.

European Commisssion - Joint Research Centre - Institute for Enviroment and Sustainability: International Reference Life Cycle Data System (ILCD) Handbook - General guide for Life Cycle Assessment - provisions and Action Steps. First edition March 2010, EUR 24378 EN. Luxembourg. Publications Office of the European Union.

Fan, S., Zakaria S., Chia C., Jamaluddin F., Nabihah S., Liew T., Pua F 2011, Comparative studies of products obtained from solvolysis liquefaction of oil palm empty fruit bunch fibres using different solvents. Bioresource technology, 102(3), 35213526.

Fedepalma 2015, Statistical Yearbook 2015. "The Oil Palm Agroindustry in Colombia and the Word 2010 - 2014”. Bogotá.
Fedepalma 2007, "Proyecto Sectorial marco MDL para la Captura de Metano, para el desplazamiento de Combustibles Fósiles y para la cogeneración de Energía Renovable en Colombia”. Tomado de: http://www.scribd.com/doc/56103970/FedepalmaApproved-CDMValidation-Report.

Fundación Carolina. (2017). "Documentación Programa Estancias Cortas Postdoctorales 2017”. Madrid, España. Tomado de: https://www.fundacioncarolina.es.

Gamarra A.R., Herrera I., Martínez-Aedo C, Lechón Y 2016, Inventario de ciclo de vida del mixeléctrico español. Presentado en: III Simposio de la Red Española de ACV. Inventario de Ciclo de Vida. Valencia, España.

García J., Uribe M., León D 1997, "Manejo de efluentes de plantas extractoras". Boletín Técnico No. 11. Cenipalma. Bogotá, Colombia.

García M 2013, Nuevos conceptos para biorrefinerías de aceite de palma. Palmas, 34, Tomo II, 66-84.

García J.; Cardenas, M., Yañez E 2010, Generación y Uso de Biomasa en Plantas de Beneficio de Palma de Aceite en Colombia. Palmas, 31(2), 41-48.

Garrain D 2017a, Seminario Taller: "Ecodiseño, etiquetado ambiental y eficiencia energética". Centro de Investigaciones Energéticas Medioambientales y Tecnológicas - CIEMAT, Madrid, España.

Garrain D 2017b, Curso Análisis de Ciclo de Vida: Introducción al Software Simapro. Centro de Investigaciones Energéticas Medioambientales y Tecnológicas - CIEMAT, Madrid, España.

Garzón A., Pacheco A 2016, "Formulación de estrategias de mejora de consumo de agua en la producción de feijoa en la Finca Cortijo de La Merced en La Vega - Cundinamarca”. Tesis. Universidad de La Salle, Bogotá.

Gauch M 2013, Ciclo de vida del biodiésel en Colombia. Palmas, 34, Tomo II, 331-344.

Goh S., Lee, K. (2010). Palm-based biofuel refinery (PBR) to substitute petroleum refinery: An energy and emergy assessment. Renewable and Sustainable Energy Reviews, 14(9), 2986-2995.

Goh S.; Tan T., Lee K., Bhatia S 2010, Bio-ethanol from lignocellulose: Status, perspectives and challenges in Malaysia. Bioresource technology, 101(13), 4834-41.

Gómez A., Mendoza L., Rincón S 2011, Activated Carbon from Oil Palm Shells for Hot Temperature Tar Removal in Gasification Systems. Chemistry, Processing Technology \& Bio-energy Conference, PIPOC 2011. International Palm Oil Congress (p. 208). Kuala Lumpur, Malaysia: MPOB.

González A., Neira L 2017, "Implementación del procedimiento para llevar a cabo la práctica de fermentación y destilación en la planta de biocombustibles del Laboratorio de Ingeniería Ambiental y Sanitaria de la Universidad de La Salle". Tesis. Universidad de La Salle, Bogotá. 
González F., Jiménez I., Rodríguez M., Restrepo S., Gómez J 2008, "Biocombustibles de segunda generación y Biodiesel: Una mirada a la contribución de la Universidad de los Andes". Revista de Ingeniería. 28, 70-82.

González-Delgado, Á.D., Kafarov V 2011, “Microalgae based biorefinery: issues to consider". CT\&F-Ciencia, Tecnología y Futuro. $4(4), 5-22$.

Guo, J., Chong Lua A 1998, Characterization of chars pyrolyzed from oil palm stones for the preparation of activated carbons. Journal of Analytical and Applied Pyrolysis, 46(2), 113-125.

Gutiérrez F., Sánchez J., Cardona C 2009), rocess integration possibilities for biodiésel production from palm oil using ethanol obtained from lignocellulosic residues of oil palm industry. Bioresource technology, 100(3), 1227-37.

Hernández V 2015, "Evaluación técnica, económica y ambiental del aprovechamiento de residuos lignocelulósicos de cadenas agroindustriales en la producción de biofertilizantes". Tesis. Universidad Nacional de Colombia. Bogotá, Colombia.

Herrera I 2016, "Evaluación Ambiental mediante el Análisis de ciclo de vida". Primer Encuentro de la Red Temática en Sustentabilidad Energética, Medioambiente y Sociedad. Red SUMAS. Morelos, México.

Herrera I., Gamarra A., Lago C., Sahagún J. Martínez-Aedo C., Lechón Y 2016a, "Life cycle analysis of actualization of biofuels for transport in Spain". The event for LCA XVI knowledge sharing and networking in North America. American Center for Life Cycle Assessment. Charleston, SC, USA.

Herrera I., Pérez J., Gamarra A 2016b, "Análisis de ciclo de vida de la producción de etanol combustible proveniente del bagazo de caña de azúcar". Presentado en: III Simposio de la Red Española de ACV (esLCA). Valencia (España).

INCONTEC, AENE Consutoría S.A 2003, "Formulación de un programa básico de normalización para aplicaciones de energía alternativa" - Guía para la implementación de sistemas de producción de biogás. Unidad de Planeación Minero Energética UPME, Bogotá.

Kelly-Yong T., Lee K., Mohamed A., Bhatia S 2007, Potential of hydrogen from oil palm biomass as a source of renewable energy worldwide. Energy Policy, 35(11), 5692-5701.

Loboguerrero C 2007, "Exploración de la factibilidad del aprovechamiento de los residuos de la extracción de aceite de palma para convertirlos en azúcares fermentables". Tesis. Universidad de los Andes. Bogotá.

Lua C., Lau Y., Guo J 2006, Influence of pyrolysis conditions on pore development of oil-palm-shell activated carbons. Journal of Analytical and Applied Pyrolysis, 76(1-2), 96-102.

Mansoornejad B., Chambost V., Stuart P 2010, Integrating product portfolio design and supply chain design for the forest biorefinery. Computers \& Chemical Engineering, 34(9), 1497-1506.
Martínez D 2017, "Evaluación de la sostenibilidad de tecnologías emergentes de producción de biocombustibles en Colombia aplicando criterios técnicos, ambientales y económicos”. Tesis. Universidad Industrial de Santander. Bucaramanga.

Mercado G., Herrera I 2017, “Análisis de ciclo de vida comparativo de un biomaterial”. Colección de documentos CIEMAT, Ed. CIEMAT. Madrid, España.

Moncada J., Cardona A., Rincón L 2015, Design and analysis of a second and third generation biorefinery: the case of castorbean and microalgae. Bioresource Technology. 198, 836-843.

Mora A., Mendoza S 2017, "Determinación de impactos ambientales en la finca Cascajal en Pacho Cundinamarca, mediante el balance de carbono y nutrientes para un cultivo de café". Tesis. Universidad de La Salle, Bogotá.

Mosquera M., Valderrama M., Fontanilla C., Ruíz E., Uńate M., Rincón F., \& Arias N 2016, Costos de producción de la agroindustria de la palma de aceite en Colombia en 2014 (2016). Palmas, 37(2), 37-53.

Muñoz M., Herrera I 2017, "Análisis de ciclo de vida de la producción de aceite vegetal de jatropha. Caso de estudio: Ecuador". Colección de Informes Técnicos CIEMAT, Ed. CIEMAT. Madrid, Espańa.

Muñoz M., Iglesias E., Herrera I. (2016). “Análisis de la decisión de los productores de jatropha para participar en el proyecto "Piñón para Galápagos" Caso de estudio Ecuador". Segundo Encuentro de la Red Temática en Sustentabilidad Energética, Medioambiente y Sociedad. Red SUMAS. Morelos, México.

Naranjo C 2017, "Seminario Taller Análisis de Ciclo de Vida”. Pacto Global Red Colombia. Bogotá, Colombia.

Nieto C., Piñeros J 2016, "Evaluación de impactos ambientales generados por los gases efecto invernadero, en la producción de pasto forrajero (Brachiaria brizantha), en la Finca Camoita, San Martín (Meta)". Tesis. Universidad de La Salle, Bogotá.

National Renewable Energy Laboratory - NREL 2008, Modeling tomorrow's biorefinery the NREL Biochemical Pilot Plant. Energy Efficiency and Renewable Energy. U.S. Department of Energy. Retrieved from http://www.nrel.gov/docs/fy08osti/41334.pdf

Ortiz R.; Fernández O 2000, "El cultivo de la palma aceitera”. Editorial Universidad Estatal a Distancia. San José, Costa Rica. 208p.

Pérez, A., Camargo, M., Rincón, P. y Marchant, M 2017, Key challenges and requirements for sustainable and industrialized biorefinery supply chain design and management: a bibliographic analysis. Renewable Sustainable Energy Reviews. 69, 350-359.

Pinzón W., Palacios D 2016, "Determinación de la huella hídrica en el cultivo de palma de aceite en la empresa Guaicaramo S.A. localizada en Barranca de Upía-Meta”. Tesis. Universidad de La Salle. Bogotá. 
Rojas L 2011, "Evaluación de pre-tratamientos biológicos y térmicos previos a la hidrólisis enzimática de fibra prensada de palma, para la producción de azúcares fermentables". Tesis. Universidad Nacional de Colombia, Bogotá.

Shinoj, S.; Visvanathan, R.; Panigrahi, S. y Kochubabu, M 2011, Oil palm fiber (OPF) and its composites: A review. Industrial Crops and Products, 33(1), 7-22.

Shuit S., Tan T., Lee K., Kamaruddin H 2009, Oil palm biomass as a sustainable energy source: A Malaysian case study. Energy, 34(9), 1225-1235.

Singh P., Ibrahim H., Esa N., Iliyana S 2010, "Composting of waste from palm oil mill: a sustainable waste management practice". Reviews in Environmental Science and Bio/Technology, 9(4), 331-344.

Tan H., Lee K., Mohamed A 2010, "Second-generation bio-ethanol (SGB) from Malaysian palm empty fruit bunch: energy and exergy analyses". Bioresource technology, 101(14), 5719-27.

Tan J., Jahim J., Harum S., Wu T., Mumtaz T 2016, "Utilization of oil palm fronds as a sustainable carbon source in biorefineries". International Journal of Hydrogen Energy, 41, 4896-4906.

Tantitham, S.; Khlaisombat, P.; Clendon, J. H.; Campbell-Board, M. y McIntosh, B 2009, "A Review of Three CDM Biogas Projects Based on Palm Oil Mill Effluent in Southern Thailand". Proceedings of the PIPOC 2009 International Palm Oil Congress (Chemistry \& Technology). Kuala Lumpur.

Torres J 2010, “Maquinaria usada para la elaboración de surfactantes anionicos derivados del aceite de palma”. Publicaciones e Investigación. 4, 147-184.

Torres J 2012, "Sulfonation/Sulfation processing technology for anionic surfactant manufacture". Advances in Chemical Engineering, Dr Zeeshan Nawaz (Ed.). InTech. Rijeka, Croatia. Available from: https://www.intechopen.com/books/advancesin-chemical-engineering/sulfonation-sulfation-processing-technology-for-anionic-surfactant-manufacture.

Torres J 2014, "Plan de manejo ambiental para una planta de biodiesel". Libro de trabajos de investigación en biocombustibles. Ediciones Universidad Industrial de Santander. Bucaramanga, Colombia.

Torres J., Díaz, A., Sánchez F 2009a , "Reactor de película descendente para la sulfonación de ésteres metílicos con trióxido de azufre”. Ingeniería e Investigación. 29(3), 48-53.
Torres J., Fonseca O. y Lizarazo J 2016, "Determinación de la huella hídrica del cultivo de algodón”. Publicaciones e Investigación. $10,39-46$.

Torres J., Herrera I 2017, "Modelo de cálculo para ACV de un cultivo de palma africana. En: Energías renovable y materiales para nuevas tecnologías en Colombia”. Varios Autores. ISBN: 978-958-8819-63-1. Ediciones UIS. Bucaramanga, Colombia, pp: 71.

Torres J., Maldonado M 2011, "Evaluación del plan de manejo ambiental para una planta de producción de biodiesel”. Publicaciones e Investigación. 5, 67-78.

Torres J., Palacios O., Sánchez F., Morales M 2009b, Mathematical model of a falling film reactor for methyl ester sulfonation. Chemical Product and Process Modeling. 4(5), Article 12, 21p.

Torres J., Sánchez E., Villareal J 2015, “Estimación de la huella hídrica para un cultivo de pitahaya amarilla" (Selenicereus megalanthus). Publicaciones e Investigación. 9, 145-156.

Torres J., Uribe S., López V 2013, “Obstáculos a la formación de una actitud investigativa”. Educación, Pedagogía y Sociedad. Editorial Redipe. Tomo 9, 161-168. Cali, Colombia.

UIS - IDEAM 1999, Sistema de información Ambiental para sectores productivos: Producción de aceites vegetales sin refinar. Bucaramanga, Colombia.

UNIPALMA S.A 2016, "Plan de Gestión Integral de Residuos" (PGIRS). 66p.

Vides A., Vides C., Ojeda E., Herrera I 2017, Evaluación ambiental de tecnologías para la generación de electricidad mediante fuentes de energía renovables en zonas aisladas de La Guajira, Colombia. Colección de documentos CIEMAT, Ed. CIEMAT. Madrid, España.

Vijayendran, B 2010, "Bio products from biorefineries - trends, challenges and opportunities". Journal of Business Chemistry, 7(3), 109-115. Retrieved from http://www.businesschemistry. org/article/?article $=121$.

Wright, M. y Brown, R. C 2007, "Establishing the optimal sizes of different kinds of biorefineries". Biofuels, Bioproducts and Biorefining, 1(3), 191-200.

Yamada H., Tanaka R., Sulaiman O., Hashim R., Hamid A., Yahya K., Kosugi A 2010, "Old oil palm trunk: A promising source of sugars for bioethanol production". Biomass and Bioenergy, 34(11), 1608-1613. 


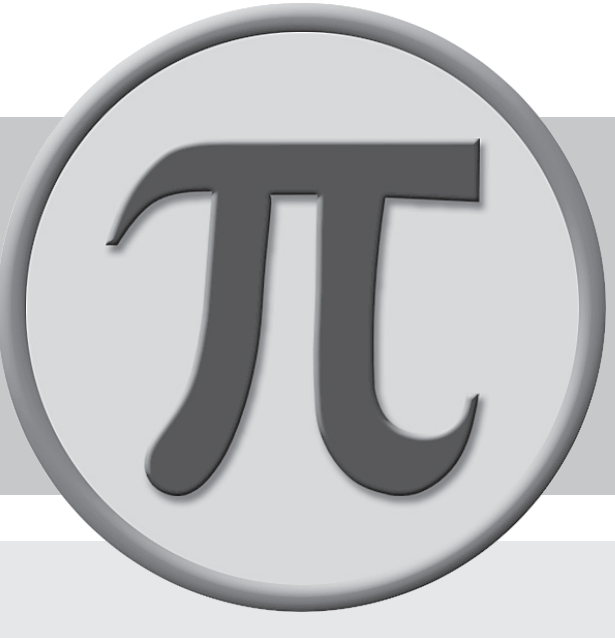


